\title{
Systemic onset juvenile idiopathic arthritis: a single center experience
}

\author{
Erdal Sağ ${ }^{1}$, Berna Uzunoğlu², Fatma Bal², Hafize Emine Sönmez ${ }^{1}$, Selcan Demir ${ }^{1}$, \\ Yelda Bilginer ${ }^{1}$, Seza Özen ${ }^{1}$ \\ ${ }^{1}$ Division of Pediatric Rheumatology, ${ }^{2}$ Department of Pediatrics, Hacettepe University Faculty of Medicine, Ankara, Turkey. \\ E-mail: sezaozen@hacettepe.edu.tr \\ Received: 5th July 2018, Revised: 18th October 2018, 20th March 2019, Accepted: 19th April 2019
}

SUMMARY: Sağ E, Uzunoğlu B, Bal F, Sönmez HE, Demir S, Bilginer Y, Özen S. Systemic onset juvenile idiopathic arthritis: a single center experience. Turk J Pediatr 2019; 61: 852-858.

Systemic juvenile idiopathic arthritis (sJIA) presents with prolonged fever and systemic features such as arthritis, rash, lymphadenopathy, hepatosplenomegaly and serositis. In this study, we aimed to evaluate the clinical and laboratory findings, and outcomes of sJIA patients from a tertiary rheumatology center.

Between 2010-2017, patients who had been diagnosed with sJIA, participated in the study. The demographics, clinical and laboratory features, and outcomes, were evaluated retrospectively.

Seventy-five sJIA (\%56 male) patients were enrolled. The mean age at diagnosis was $6,45 \pm 4,80$ years. At the time of diagnosis, the most common findings were fever (\%100) followed by arthritis $(78,7 \%)$, and rash $(66,2 \%)$. Twenty-four percent of the patients present with macrophage activation syndrome (MAS) at the time of diagnosis. Totally, $36 \%$ of the patients had at least one MAS attack during the course of the disease. $46 \%$ of the patients had polyphasic course while $54 \%$ had one attack ( $26 \%$ monophasic, $28 \%$ persistant). All of the patients were treated with non-steroid anti-inflammatory drugs (NSAID) and/or corticosteroids at the beginning of the disease. Twenty percent of the patients reached remission with corticosteroid or disease-modifying anti-rheumatic drugs (DMARDs) however the rest of the patients needed at least one biologic agent. Anakinra was the most common first-line biologic treatment choice $(n=45)$. Fourteen $(18,7 \%)$ of the patients had polyarticular joint involvement during the disease course, and 5 of them achieved remission with tocilizumab.

Systemic JIA is an important disease with high risk of morbidity and mortality. As our center is one of the most important tertiary referral rheumatology centers in the country, we had a high MAS incidence. Eighty percent of the patients achieved remission with a biological agent. Anti-IL1 drugs are mostly preferred for ongoing systemic inflammation. Anti-IL-6 agents are very efficient in patients with a polyarticular course.

Key words: systemic juvenile idiopathic arthritis, sJIA, macrophage activation syndrome, MAS, sJIA treatment.

Systemic juvenile idiopathic arthritis (sJIA) is characterized by a potentially severe course, including prolonged fever, systemic features, and destructive arthritis. The most devastating complication of sJIA is macrophage activation syndrome (MAS).

Patients are classified as sJIA according to International League of Associations for 
Rheumatology (ILAR) criteria with the presence of a documented quotidian fever of at least 2 weeks duration and arthritis; plus one of the following: typical rash, generalized lymphadenopathy, hepatomegaly or splenomegaly, or serositis. ${ }^{1}$ In Europe, sJIA accounts for $5-15 \%$ of all children with chronic arthritis with an annual incidence of 0.3-0.8 cases per 100,000 children under 16 years of age. ${ }^{2}$ Approximately $10 \%-40 \%$ of sJIA patients present a monocyclic disease course, recovering completely within 12-24 months. ${ }^{3,4}$ However, more than half of the children with sJIA have a chronic disease course and a minority of patients follow polycyclic course with an episode of inactive period of disease without any medications. ${ }^{3,4}$ With recent insights into the pathogenesis, biologic therapies have enabled us to provide more targeted treatment.

In this study, our aim was to present the clinical and laboratory findings, treatment responses and outcomes of sJIA patients from a tertiary rheumatology center.

\section{Material and Methods}

Systemic JIA patients, who had been diagnosed and were being followed at the Department of Pediatric Rheumatology of Hacettepe University between January 2010 and January 2017, were included in this study. The patients were classified as having sJIA according to ILAR criteria. ${ }^{1}$ Patient files were analyzed retrospectively for clinical findings, laboratory features and outcome results.

MAS was diagnosed according to the criteria defined by Ravelli et al. ${ }^{5}$ (fever, ferritin $>684$ $\mathrm{ng} / \mathrm{dl}$, and any 2 of the following: platelet count $<181 \times 10^{9} / \mathrm{L}$, aspartate aminotransferase $($ AST $)>48 \mathrm{U} / \mathrm{L}$, triglycerides $>156 \mathrm{mg} / \mathrm{dl}$, fibrinogen $<360 \mathrm{mg} / \mathrm{dl}$ ).

Remission (inactive disease activity) was defined as lack of fever, rash, serositis, splenomegaly, lymphadenopathy, and arthritis, as well as normal levels of erythrocyte sedimentation rate (ESR) and C-reactive protein (CRP). ${ }^{6}$ This study was approved by the Ethics Committee of Hacettepe University. (September 12, 2017; GO 17/743-37)

\section{Statistical analysis}

SPSS software version 21 was used to evaluate the statistical analysis. The variables were investigated using visual (histogram, probability plots) and analytic methods (Kolmogorov-Smirnov) to determine whether or not they are normally distributed. The data of descriptive analysis were expressed as the median, minimum, and maximum values. The Mann-Whitney $U$ test was used to compare the non-normally distributed continuous data between two groups. $\mathrm{p}<0.05$ was considered as statistically significant.

\section{Results}

Seventy-five sJIA patients were included into this study. Fifty-six percent of the patients were male. The mean age at diagnosis was 6.45 \pm 4.80 years. At the time of diagnosis all of the patients had typical fever while $78.7 \%$ of them had arthritis, $66.2 \%$ of them had rash, $37.3 \%$ of them had arthralgia, $28.4 \%$ of them had hepatosplenomegaly (HSM), $20.3 \%$ of them had lymphadenopathy, and $17.6 \%$ of them had serositis (Fig. 1). Twenty-four percent of the patients had MAS at the time of diagnosis while in total $36 \%$ of the patients had at least one MAS attack anytime on the disease course. As to the course of the disease; $46 \%$ of the patients had recurrent attacks however $54 \%$ had one attack (26\% monophasic, $28 \%$ persistent). Fourteen (18.7\%) of the patients had polyarticular joint involvement during the disease course. There was no significant difference between the patients with

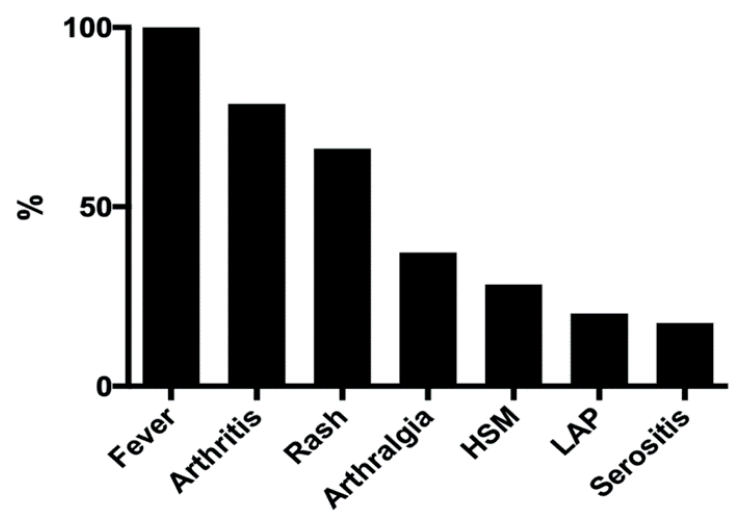

Fig. 1. Frequency of ILAR criteria at the time of diagnosis. 
polyphasic and monophasic course in terms of laboratory and clinical features except for the presence of HSM which was more common in patients with polyphasic course $(42.4 \%$ vs $17.1 \% ; \mathrm{p}=0.016$, respectively). Thirty-two patients had flares during the disease course. Ten of them had flares under certain treatment and 22 of them had flares after treatment cessation. Mean interval between flare and the last remission was 20 months.

Sixteen patients $(21.3 \%)$ presented with systemic features other than arthritis. When we compared this group with the patients who presented with arthritis; serositis $(40 \%$ vs $12 \%$; p: 0.011$)$ and rash $(93.3 \%$ vs $59.3 \%$ p:0.013) were more common. MAS was more common at both presentation (40\%vs $21 \%$ ) and during the disease course (50\% vs $32.2 \%$ ) however these differences were not significant. Among these 16 patients, 10 of them had a monophasic, 6 of them had a polyphasic course. Two of the patients had polyarticular involvement during the follow-up period.

When we compared the patients who had presented with MAS at the time of diagnosis to the others, they were older than other sJIA patients (mean $8.9 \pm 4.7$ vs $5.3 \pm 4.2$ years; $\mathrm{p}=0.004)$ with a male predominance $(83.3 \%$ vs $47.3 \%$ : p: 0.009) (Table I). The patients with
MAS also had a lower WBC (mean 7166 \pm 5637 / $\mathrm{mm}^{3}$ vs $\left.17864 \pm 9579 / \mathrm{mm}^{3} ; \mathrm{p}<0.001\right)$ and platelet (mean $179666 \pm 160745 / \mathrm{mm}^{3}$ vs $\left.689148 \pm 98819 / \mathrm{mm}^{3} ; \mathrm{p}<0.001\right)$ count, and had a lower erythrocyte sedimentation rate (mean $12 \pm 4.2 \mathrm{~mm} /$ hourvs $63 \pm 27.5 \mathrm{~mm} /$ hour; $\mathrm{p}<0.001)$ as expected. The patients with MAS at the time of diagnosis had serositis $(44.4 \%$ vs $7.5 \%$; $\mathrm{p}<0.001)$ and HSM $(55.6 \%$ vs $18.9 \%$; $\mathrm{p}=0.004)$ more frequently, however there was no significant difference in terms of arthritis, arthralgia, rash and lymphadenopathy. (Fig. 2.)

All of the patients were treated with nonsteroid anti-inflammatory drugs (NSAIDs) or corticosteroids at the beginning of the disease. Twenty percent $(n=15)$ of the patients reached remission within mean $9.8 \pm 7.7$ months with corticosteroid or disease-modifying antirheumatic drugs (DMARDs) however rest of the patients needed at least one biologic drug.

Anti-IL1 agents were the most common firstline biologic treatment of choice $(n=45)$ in patients not responding to conventional treatment. The treatment of 15 patients were switched to tocilizumab due to polyarticular course or lack of response to anti-IL1 treatment. Forty-six patients treated with biologic drugs achieved remission within a
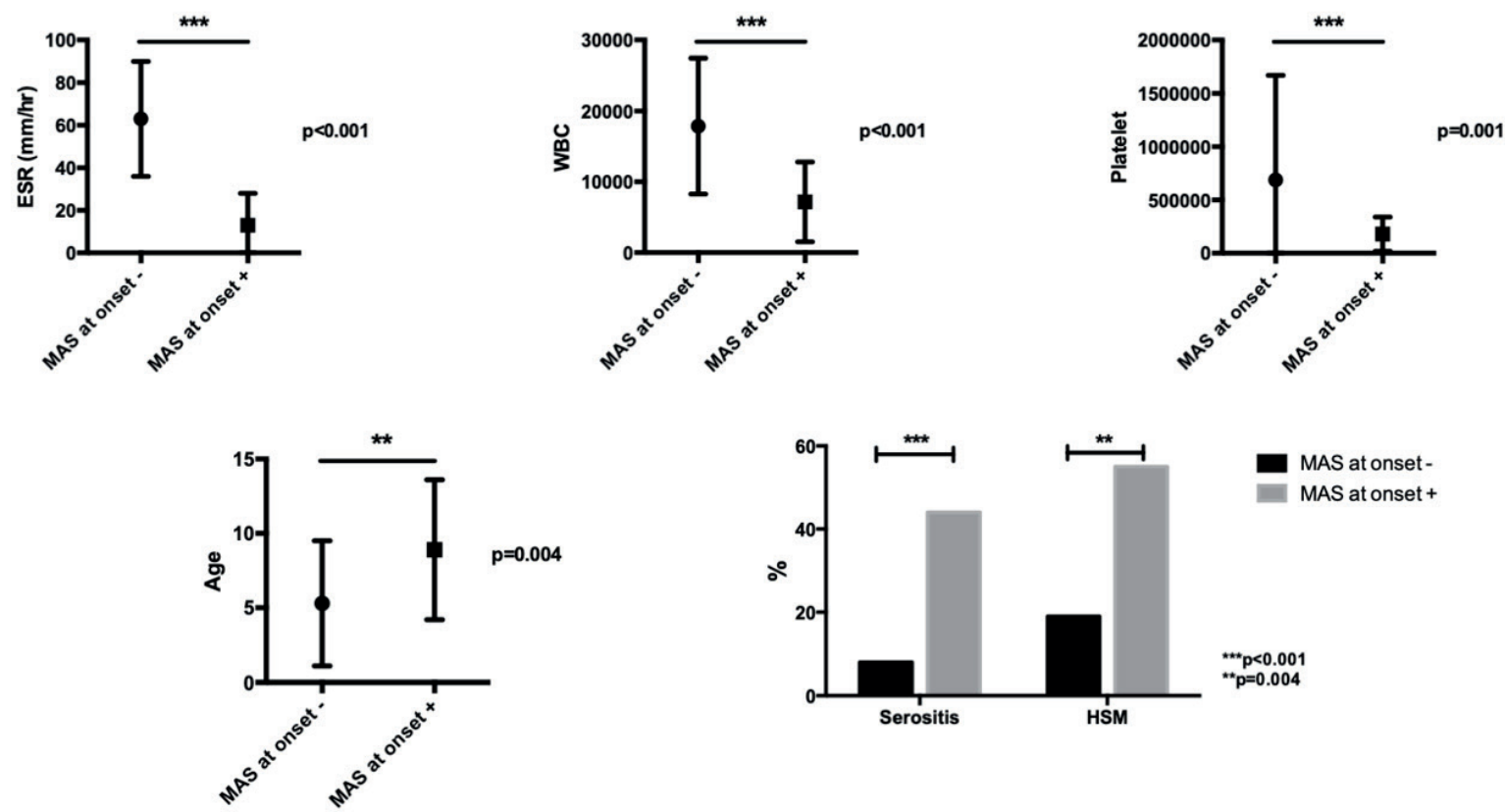

MAS: Macrophage activating syndrome WBC: White blood cell

Fig. 2. Clinical and laboratory differences between the patients presenting with MAS and the others. 


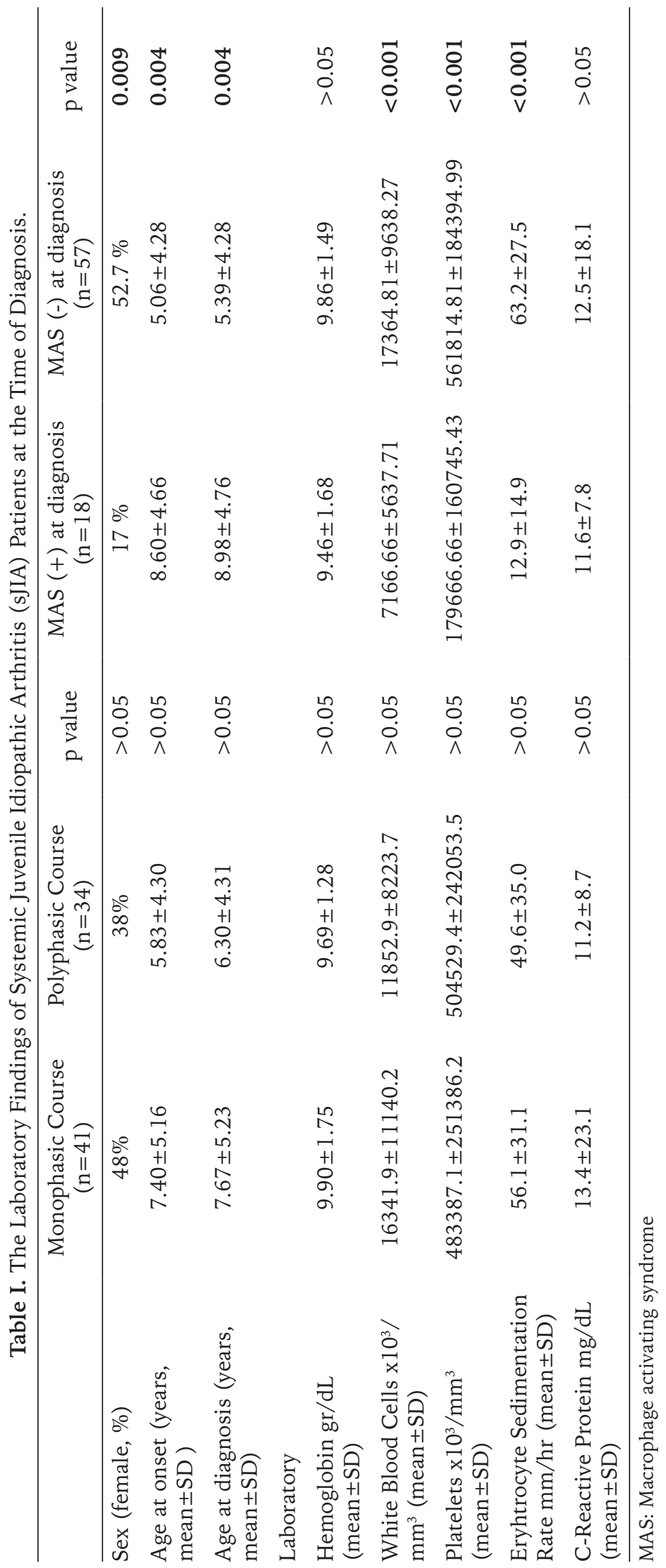




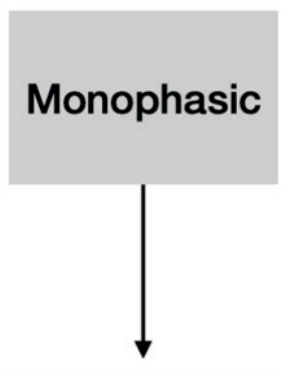

22 Anti-IL1

(19 Anakinra, 3 Canakinumab)

3 Tocilizumab

1 Etanercept

9 Corticosteroid/ DMARDs

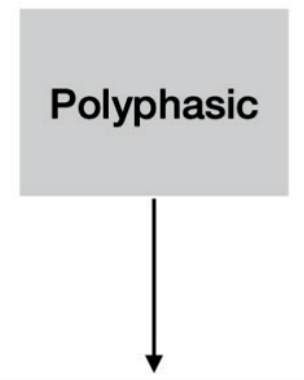

20 Anti-IL1

(14 Anakinra, 6 Canakinumab)

5 Tocilizumab

1 Etanercept

6 Corticosteroid/ DMARDs

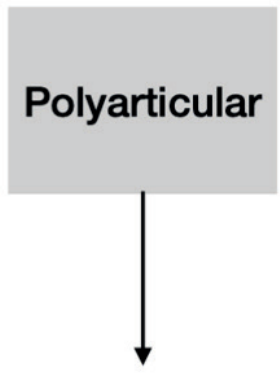

- 5 Tocilizumab

- 4 Anakinra

- 1 Etanercept

- 1 Corticosteroid/

DMARDs

*Patients with remission ( $n=67$ ) represented only. 8 patients have active disease.

**Among polyarticular group with remission, 4 patients had monophasic, 7 patients had polyphasic course, 3 patients have active disease

DMARDs: disease-modifying anti-rheumatic drugs

Fig. 3. Treatment sustaining remission in different sJIA subgroups.

median 19.7 (range 0-54.9) months. Among the patients whom remission was achieved, $44 \%$ of them were treated with anakinra, $12 \%$ with canakinumab, $10.7 \%$ with tocilizumab (Fig. 3).

Five out of 8 patients who had polyarticular joint involvement were treated with tocilizumab and achieved remission. All of these 8 patients were treated first with etanercept then were switched to anakinra, subsequently they were switched to tocilizumab due to lack of response to the above.

At follow-up, $40 \%$ of the patients $(n=30)$ are being followed without any treatment, $49 \%$ of them $(n=37)$ with treatment under remission. Eight of our patients still have active disease.

\section{Discussion}

Systemic JIA is an important disease with high risk of morbidity and mortality. In this study, we summarized the clinical and laboratory characteristics of sJIA patients. The main findings of our study are as follows: a majority of the patients presented with polyphasic or chronic ( $46 \%$ vs $28 \%$ ) course and approximately one third of the patients $(36 \%)$ had at least one MAS attack anytime during their disease course. Furthermore, $18.7 \%$ of the patients developed polyarticular joint involvement during the disease course.

Children with sJIA may present a monocyclic, polycyclic or chronic course. About $10 \%-40 \%$ of sJIA patients are reported to have a monocyclic disease course, while approximately half of sJIA patients SJIA present a chronic disease. ${ }^{3,4}$ In contrast to previous studies, $46 \%$ of our patients had polyphasic, $26 \%$ had monophasic, $28 \%$ had chronic disease course.

MAS may occur in $7-13 \%$ of sJIA patients, however evidence of subclinical MAS at the diagnosis may be found in approximately half of sJIA patients. ${ }^{7,8}$ As our center is one of the most important referral rheumatology centers in the country, we expect to see higher MAS incidence than the previously reported sJIA series (\%34). In the largest multicenter study describing the characteristics of 362 pediatric MAS patients complicating sJIA, $96 \%$ had fever, $70 \%$ hepatomegaly, and $58 \%$ splenomegaly. ${ }^{9}$ In our cohort $24 \%$ of our patients presented with MAS at the time of diagnosis. These group of patients, manifesting MAS at the time of diagnosis, were older, had serositis and HSM more frequently when compared to the rest of the cohort. Anakinra was administered to all of these patients. 
Not all of the patients with sJIA present with arthritis. In our cohort about 1 out of 5 patients presented with prominent systemic features other than arthritis. Differential diagnosis is more difficult in this group as there is no arthritis but fever, rash, serositis and other systemic features. Although the difference was not significant, it is very important to note that this group of patients are more prone to have MAS, which can be highly mortal if it is not diagnosed early.

Corticosteroids and the DMARDs have been historically the first line therapies in sJIA. Sura et al. ${ }^{10}$ have demonstrated clinical inactive disease in $25.5 \%$ of the sJIA patients with NSAIDs. In our study, $20 \%$ of the patients achieved remission with NSAID, corticosteroid or DMARDs, while the rest of the patients needed at least one biologic drug. According to the American College of Rheumatology (ACR) recommendations in sJIA treatment, those with predominantly systemic findings and moderate-severe disease activity may be treated with biologic drugs such as anti-IL1. In sJIA patients with predominantly joint involvement and moderate-severe disease activity despite three months methotrexate, treatment may be switched to anti-IL 1 or antiIL $6 .{ }^{11}$ Biological agents were very beneficial treatment in $80 \%$ of the sJIA patients in our cohort, however $1 / 5$ of our patients achieved remission without any biological agent. AntiIL1 drugs are the mostly preferred treatment choice in patients with ongoing systemic inflammation while anti interleukin-6 (antiIL-6) agents are very efficient in patients with polyarticular course.

Schneider et al. ${ }^{12}$ have showed that, before the biologic era, approximately one third of the patients developed destructive polyarticular arthritis within 5 years. In our study, $18.7 \%$ of the patients $(n=14)$ had a polyarticular course. Among them 11 patients reached remission while 5 of them achieved remission with tocilizumab. Thus, it can be speculated that anti IL-6 treatment is very beneficial in this specific group of patients.

The major limitations of our study were the retrospective design with a relatively small sample size from a single medical center.
Systemic JIA is an important disease with high risk of morbidity and mortality. As our center is one of the most important tertiary referral rheumatology centers in the country, we had a high MAS incidence. Eighty percent of the patients achieved remission with a biological agent. Anti-IL1 drugs are the mostly preferred for ongoing systemic inflammation. Anti-IL-6 agents are very efficient in patients with a polyarticular course.

\section{REFERENCES}

1. Petty RE, Southwood TR, Manners P, et al; International League of Associations for Rheumatology International League of Associations for Rheumatology classification of juvenile idiopathic arthritis: second revision, Edmonton, 2001. J Rheumatol 2004; 31: 390-392

2. Shenoi S, Horneff G, Cidon M, et al. The burden of systemic juvenile idiopathic arthritis for patients and caregivers: an international survey and retrospective chart review. Clin Exp Rheumatol 2018; 36: 920-928.

3. Singh-Grewal D, Schneider R, Bayer N, Feldman $\mathrm{BM}$ Predictors of disease course and remission in systemic juvenile idiopathic arthritis: significance of early clinical and laboratory features. Arthritis Rheum 2006; 54: 1595-1601.

4. Lovell DJ, Passo M, Giannini E, Brunner H. Systemic onset juvenile idiopathic arthritis: a retrospective study of 80 consecutive patients followed for 10 years. J Rheumatol 2001; 28: 220.

5. Ravelli A, Minoia F, Davi S, et al; Paediatric Rheumatology International Trials Organisation; Childhood Arthritis and Rheumatology Research Alliance; Pediatric Rheumatology Collaborative Study Group; Histiocyte Society. 2016 Classification Criteria for Macrophage Activation Syndrome Complicating Systemic Juvenile Idiopathic Arthritis: A European League Against Rheumatism/American College of Rheumatology/Paediatric Rheumatology International Trials Organisation Collaborative Initiative. Ann Rheum Dis 2016; 75: 481-489.

6. Wallace CA, Giannini EH, Huang B, Itert L, Ruperto $\mathrm{N}$; Childhood Arthritis Rheumatology Research Alliance, Pediatric Rheumatology. Collaborative Study Group; Paediatric Rheumatology International Trials Organizsation. American College of Rheumatology provisional criteria for defining clinical inactive disease in select categories of juvenile idiopathic arthritis. Arthritis CareRes (Hoboken) 2011; 63: 929936

7. Behrens EM, Beukelman T, Paessler M, Cron RQ Occult macrophage activation syndrome in patients with systemic juvenile idiopathic arthritis. J Rheumatol 2007; 34: 1133-1138. 
8. Boom V, Anton J, Lahdenne P, et al. Evidence-based diagnosis and treatment of macrophage activation syndrome in systemic juvenile idiopathic arthritis. Pediatr Rheumatol Online J 2015; 13: 55.

9. Minoia F, Davi S, Horne A, et al; Pediatric Rheumatology International Trials Organization; Childhood Arthritis and Rheumatology Research Alliance; Pediatric Rheumatology Collaborative Study Group; Histiocyte Society. Clinical features, treatment, and outcome of macrophage activation syndrome complicating systemic juvenile idiopathic arthritis: a multinational, multicenter study of 362 patients. Arthritis Rheumatol 2014; 66: 3160-3169.

10. Sura A, Failing C, Sturza J, Stannard J, Riebschleger M. Patient characteristics associated with response to NSAID monotherapy in children with systemic juvenile idiopathic arthritis. Pediatr Rheumatol Online J 2018; 16: 2.
11. Ringold S, Weiss PF, Beukelman T, et al. 2013 update of the 2011 American College of Rheumatology recommendations for the treatment of juvenile idiopathic arthritis: recommendations for the medical therapy of children with systemic juvenile idiopathic arthritis and tuberculosis screening among children receiving biologic medications. Arthritis Rheum 2013; 65: 2499-2512.

12. Schneider R, Lang BA, Reilly BJ, et al. Prognostic indicators of joint destruction in systemic-onset juvenile rheumatoid arthritis. J Pediatr 1992; 120 (2 Pt 1): 200-205. 